\title{
Equation of state of hypernuclear matter: impact of hyperon-scalar-meson couplings
}

\author{
Giuseppe Colucci and Armen Sedrakian \\ Institute for Theoretical Physics, J. W. Goethe University, D-60438 Frankfurt-Main, Germany
}

\begin{abstract}
We study the equation of state and composition of hypernuclear matter within a relativistic density functional theory with density-dependent couplings. The parameter space of hyperon-scalar-meson couplings is explored by allowing for mixing and breaking of SU(6) symmetry, while keeping the nucleonic couplings constant fixed. The subset of equations of state, which corresponds to small values of hyperon-scalar-meson couplings allows for massive $M \leq 2.25 M_{\odot}$ compact stars; the radii of hypernuclear stars are within the range $12-14 \mathrm{~km}$. We also study the equation of state of hot neutrino-rich and neutrinoless hypernuclear matter and confirm that neutrinos stiffen the equation of state and dramatically change the composition of matter by keeping the fractions of charged leptons nearly independent of the density prior to the onset of neutrino transparency. We provide piecewise polytropic fits to six representative equations of state of hypernuclear matter, which are suitable for applications in numerical astrophysics.
\end{abstract}

PACS numbers: 97.60.Jd,26.60.Kp,14.20.Jn,26.60.-c

\section{INTRODUCTION}

The integral parameters of compact stars depend on their equation of state (hereafter EoS) at high densities. Measurements of pulsar masses in binaries provide the most valuable information on the underlying EoS because these, being deduced from binary system parameters, are model independent within a given theory of gravity [1]. The recent discovery of the pulsar J1614-2230 in a binary orbit with a white dwarf provided the initial evidence for $2 M_{\odot}$ compact stars. The mass of the compact star, measured via the Shapiro delay, is $1.97 \pm 0.04 M_{\odot}$ [2]. The recent observation of a relativistic binary consisting of a white dwarf and a pulsar (J0348+0432) in optical and radio bands, respectively, provided another measurement, with similar accuracy and slightly larger mass $2.01 \pm 0.04 M_{\odot}$ 3]. The fact that the masses were measured by different methods strengthens the idea that massive $\left(2 M_{\odot}\right)$ compact stars exist in nature. The first of these observations spurred an intensive discussion of the phase structure of dense matter, which is consistent with the implied observational lower bound on the maximum mass of any sequence of compact stars [4 27].

Pulsar radii have been extracted, e.g., from modeling the x-ray binaries under certain reasonable model assumptions, but the uncertainties are large 28 31]. An example that we use in our analysis, is the pulse phaseresolved x-ray spectroscopy of PSR J0437-4715, which sets a lower limit on the radius of a $1.76 M_{\odot}$ solar mass compact star $R>11 \mathrm{~km}$ within $3 \sigma$ error 31 .

Large masses and radii are evidence for the relative stiffness of the EoS of dense matter at high densities. Large densities may require substantial population of heavy baryons (hyperons), because these become energetically favorable once the Fermi energy of neutrons becomes of the order of their rest mass. Their onset then reduces the degeneracy pressure of a cold thermodynamic ensemble and softens effectively the EoS of dense matter. This decreases the maximum mass of a compact stars to values which contradict the observation of massive com- pact stars in nature. This controversy between the theory and observations is the essence of the "hyperonization puzzle" in compact stars.

Although the emergence of heavy baryons (mainly $\Sigma^{ \pm}$ and $\Lambda$ hyperons) were considered even before the discovery of pulsars and their identification with the neutron stars [32], their existence in the cores of neutron stars is still elusive. Seminal work on interacting hyperonic matter was carried early after the discovery of pulsars 33, 34]. Systematic studies of interacting dense hypernuclear matter were carried with the advent of relativistic density functional methods [35 44]; for reviews see Refs. [45, 46]. Most of these works predicted masses that are not much larger than the canonical mass of a neutron star (in contradiction with modern observations). Masses on the order of $\leq 1.8 M_{\odot}$ were obtained later in non-relativistic phenomenological models [47]. Fully microscopic models based on hyperonnucleon potentials, which include the repulsive threebody forces, predict low maximal masses for hypernuclear stars [48, 49].

Finite-temperature hyperonic matter in the presence of a thermal bath of neutrinos may differ significantly from the cold hypernuclear matter found in evolved neutron stars. Previous studies [50 53] showed that as the EoS becomes stiffer, the deleptonizing effect of charged hyperons at zero temperature is removed in the presence of neutrinos and the sequence in which hyperons appear changes.

In this work we contribute to the investigation of the "hyperonization puzzle" by studying the parameter space of the coupling of hyperons to the light scalar octet of mesons. The parameter space is partially motivated by the hypernuclear potentials as well as investigations of the external field QCD sum rules that involve SU(6) symmetry breaking and mixing effects [54, 55]. Because the structure and even content of the scalar mesons is still a subject of discussion it appears necessary to investigate this particular domain of hypernuclear physics with respect to the consistency with the compact star obser- 
vations. Conversely, as we show below, the considered parameter space can in fact account for massive hyperonic compact stars, thus providing a possible solution to the "hyperonization puzzle".

To study the EoS of hypernuclear matter we use a model based on relativistic density functional theory of Walecka and a density-dependent parametrization of the nucleon-meson couplings of Ref. [56]. The EoS and composition of matter are studied both in the zerotemperature limit relevant to mature compact stars as well as at finite temperatures relevant for the hot protoneutron star stage of evolution. In the latter case we assume neutrino-rich matter in $\beta$ equilibrium and explore the effects of finite temperature and neutrino content on the stiffness and composition of matter. We confirm the general features found in the previous analysis 50 53. and provide a detailed account of these effects within our model.

This paper is structured as follows. In Sec. II we discuss the theoretical set-up of the relativistic density functional theory. The parametrization of the coupling constant of the theory is discussed in Sec. III] Section IV] is devoted to the discussion of the choice of the hyperonmeson coupling constants. We present our results in Sec. V] Finally, our conclusions are collected in Sec. VI]

\section{THEORETICAL MODEL}

The relevant degrees of freedom in nuclear matter at high density are nucleons and hyperons. In particular, we choose as degrees of freedom of our relativistic mean field model the baryon octet $\left(J^{P}=\frac{1}{2}^{+}\right)$, and we study the interaction of these fields with the isoscalar-scalar, $\sigma$, isoscalar-vector, $\omega_{\mu}$, and isovector-vector, $\boldsymbol{\rho}_{\mu}$, mesons. Besides these fields, we also consider the presence of leptons, $e^{-}, \mu^{-}$. In the case of finite-temperature matter, which describes proto-neutron star matter, the model has the neutrino degrees of freedom, because they are expected to be trapped in a proto-neutron star after the first minute of its birth. The introduction of additional variables, the neutrino chemical potentials, requires additional constraints, which we provide by fixing the lepton fractions, $Y_{L l}$, appropriate for conditions prevailing in the evolution of a proto-neutron star [51].

The relativistic Lagrangian reads

$$
\begin{aligned}
\mathcal{L} & =\sum_{B} \bar{\psi}_{B}\left[\gamma^{\mu}\left(i \partial_{\mu}-g_{\omega B} \omega_{\mu}-\frac{1}{2} g_{\rho B} \boldsymbol{\tau} \cdot \boldsymbol{\rho}_{\mu}\right)\right. \\
& \left.-\left(m_{B}-g_{\sigma B} \sigma\right)\right] \psi_{B}+\frac{1}{2} \partial^{\mu} \sigma \partial_{\mu} \sigma-\frac{1}{2} m_{\sigma}^{2} \sigma^{2} \\
& -\frac{1}{4} \omega^{\mu \nu} \omega_{\mu \nu}+\frac{1}{2} m_{\omega}^{2} \omega^{\mu} \omega_{\mu}-\frac{1}{4} \boldsymbol{\rho}^{\mu \nu} \boldsymbol{\rho}_{\mu \nu}+\frac{1}{2} m_{\rho}^{2} \boldsymbol{\rho}^{\mu} \cdot \boldsymbol{\rho}_{\mu} \\
& +\sum_{\lambda} \bar{\psi}_{\lambda}\left(i \gamma^{\mu} \partial_{\mu}-m_{\lambda}\right) \psi_{\lambda}-\frac{1}{4} F^{\mu \nu} F_{\mu \nu}
\end{aligned}
$$

where the $B$ sum is over the $J^{P}=\frac{1}{2}^{+}$baryon octet and
$\psi_{B}$ are the baryonic Dirac fields with masses $m_{B}$. The interaction is mediated by the $\sigma, \omega_{\mu}$ and $\boldsymbol{\rho}_{\mu}$ meson fields with the $\omega_{\mu \nu}$ and $\boldsymbol{\rho}_{\mu \nu}$ field strength tensors and masses $m_{\sigma}, m_{\omega}$, and $m_{\rho}$. The baryon-meson coupling constants are denoted by $g_{m B}$ (their numerical value is discussed in Sec. III below.) The $\lambda$-sum in Eq. (11) runs over the leptons $e^{-}, \mu^{-}, \nu_{e}$ and $\nu_{\mu}$ with masses $m_{\lambda}$ and the last term is the electromagnetic energy density. The contribution of neutrinos to the sum above is included only at non-zero temperature, when they are trapped in stellar matter and form a statistical ensemble in equilibrium. In mature compact stars at essentially zero temperature the neutrinos do not contribute to the thermodynamical quantities of matter because they are out of equilibrium and their chemical potential vanishes.

The Lagrangian density (11) yields the pressure and the energy density, which at finite temperature read respectively

$$
\begin{aligned}
P & =-\frac{m_{\sigma}^{2}}{2} \sigma^{2}+\frac{m_{\omega}^{2}}{2} \omega_{0}^{2}+\frac{m_{\rho}^{2}}{2} \rho_{03}^{2}+\frac{1}{3} \sum_{B} \frac{2 J_{B}+1}{2 \pi^{2}} \\
& \times \int_{0}^{\infty} \frac{d k k^{4}}{E_{k}^{B}}\left[f\left(E_{k}^{B}-\mu_{B}^{*}\right)+f\left(E_{k}^{B}+\mu_{B}^{*}\right)\right] \\
& +\frac{1}{3 \pi^{2}} \sum_{\lambda} \int_{0}^{\infty} \frac{d k k^{4}}{E_{k}^{\lambda}}\left[f\left(E_{k}^{\lambda}-\mu_{\lambda}\right)+f\left(E_{k}^{\lambda}+\mu_{\lambda}\right)\right]
\end{aligned}
$$

and

$$
\begin{aligned}
\epsilon & =\frac{m_{\sigma}^{2}}{2} \sigma^{2}+\frac{m_{\omega}^{2}}{2} \omega_{0}^{2}+\frac{m_{\rho}^{2}}{2} \rho_{03}^{2}+\sum_{B} \frac{2 J_{B}+1}{2 \pi^{2}} \\
& \times \int_{0}^{\infty} d k k^{2} E_{k}^{B}\left[f\left(E_{k}^{B}-\mu_{B}^{*}\right)+f\left(E_{k}^{B}+\mu_{B}^{*}\right)\right] \\
& +\sum_{\lambda} \int_{0}^{\infty} \frac{d k}{\pi^{2}} k^{2} E_{k}^{\lambda}\left[f\left(E_{k}^{\lambda}-\mu_{\lambda}\right)+f\left(E_{k}^{\lambda}+\mu_{\lambda}\right)\right],
\end{aligned}
$$

where $\sigma, \omega_{0}$ and $\rho_{03}$ are the nonvanishing mesonic mean fields, $J_{B}$ is the baryon degeneracy factor, $m_{B}^{*}=m_{B}-$ $g_{\sigma B}$ is the effective baryon mass, $\mu_{B}^{*}=\mu_{B}-g_{\omega B} \omega_{0}-$ $g_{\rho B} I_{3} \rho_{3}^{0}$ is the baryon chemical potential including the time component of the fermion self-energy, $I_{3}$ is the third component of baryon isospin, $E_{k}^{B}=\sqrt{k^{2}+m_{B}^{* 2}}$ and $E_{k}^{\lambda}=\sqrt{k^{2}+m_{\lambda}^{2}}$ are the single particle energies of baryons and leptons respectively, and $f(y)=[1+$ $\exp (y / T)]^{-1}$ is the Fermi distribution function with $T$ being the temperature. We take each lepton mass $m_{\lambda}$ equal to its free-space value.

At nonzero temperature the net entropy of the matter is the sum of the baryon, $S_{B}$, and lepton, $S_{L}$, contributions

$$
\begin{aligned}
S_{B} & =-\sum_{B} \frac{2 J_{B}+1}{2 \pi^{2}} \int_{0}^{\infty} d k k^{2}\left\{\left[f\left(E_{k}^{B}-\mu_{B}^{*}\right) \ln f\left(E_{k}^{B}-\mu_{B}^{*}\right)\right.\right. \\
& \left.\left.+\bar{f}\left(E_{k}^{B}-\mu_{B}^{*}\right) \ln \bar{f}\left(E_{k}^{B}-\mu_{B}^{*}\right)\right]+\left(\mu_{B}^{*} \rightarrow-\mu_{B}^{*}\right)\right\}
\end{aligned}
$$


and

$$
\begin{aligned}
S_{L} & =-\sum_{\lambda} \int_{0}^{\infty} \frac{d k}{\pi^{2}}\left[f\left(E_{k}^{\lambda}-\mu_{\lambda}\right) \ln f\left(E_{k}^{\lambda}-\mu_{\lambda}\right)\right. \\
& \left.+\bar{f}\left(E_{k}^{\lambda}-\mu_{\lambda}\right) \ln \bar{f}\left(E_{k}^{\lambda}-\mu_{\lambda}\right)\right],
\end{aligned}
$$

where $\bar{f}(y)=1-f(y)$. The free-energy density is then given by

$$
F=\epsilon-T\left(S_{B}+S_{L}\right)
$$

\section{DENSITY-DEPENDENT PARAMETRIZATION DD-ME2}

Below, we work with a density-dependent parametrization, which is designed to account for in an economical manner the many-body correlations that arise beyond the mean-field approximation. The density dependence of the coupling constant thus accounts for the influence of the medium on the scattering of baryons. We choose to work with the Density Dependent Meson Exchange (DDME2) parametrization of Ref. [56], which introduces an explicit density dependence in the nucleon-meson couplings $g_{N \sigma}, g_{N \omega}$ and $g_{N \rho}$. The phenomenological ansatz for the density dependence for the $\sigma$ - and $\omega_{\mu}$-meson coupling constants is [56, 57]

$$
g_{i N}\left(\rho_{B}\right)=g_{i N}\left(\rho_{0}\right) h_{i}(x), \quad i=\sigma, \omega,
$$

where $\rho_{B}$ is the baryon density,

$$
h_{i}(x)=a_{i} \frac{1+b_{i}\left(x+d_{i}\right)^{2}}{1+c_{i}\left(x+d_{i}\right)^{2}}
$$

is a function of $x=\rho_{B} / \rho_{0}$ and $\rho_{0}$ is the nuclear saturation density. The parameters in Eq. (8) are not independent. Indeed, the five constraints $h_{i}(1)=1, h_{\sigma}^{\prime \prime}(1)=$ $h_{\omega}^{\prime \prime}(1), h_{i}^{\prime \prime}(0)=0$ reduce the number of independent parameters to three. Three additional parameters in the isoscalar-scalar channel are $g_{N \sigma}\left(\rho_{0}\right), g_{N \omega}\left(\rho_{0}\right)$ and $m_{\sigma}$, the mass of the phenomenological $\sigma$ meson. Microscopic calculations show that the $\boldsymbol{\rho}_{\mu}$-meson coupling decreases at high densities [57]. Therefore, instead of the ansatz (8) one can use a minimalistic ansatz, with exponential form of density dependence [56, 57]

$$
g_{\rho N}\left(\rho_{B}\right)=g_{\rho N}\left(\rho_{0}\right) \exp \left[-a_{\rho}(x-1)\right] .
$$

Thus, the parametrization we use has in total eight parameters, which are adjusted to reproduce the properties of symmetric and asymmetric nuclear matter, binding energies, charge radii, and neutron radii of spherical nuclei. The parameters of the DD-ME2 effective interaction are shown in Table @

When the coupling constants are density dependent, the thermodynamical consistency (specifically the energy conservation and fulfillment of the Hugenholtz-van Hove theorem) require the inclusion of the so-called rearrangement self-energy [58]. This contributes to the pressure,
TABLE I. Meson masses and couplings to the baryons in DDME2 effective interaction.

\begin{tabular}{cccc}
\hline & $\sigma$ & $\omega$ & $\rho$ \\
\hline$m_{i}[\mathrm{MeV}]$ & 550.1238 & 783.0000 & 763.0000 \\
$g_{N i}\left(\rho_{0}\right)$ & 10.5396 & 13.0189 & 3.6836 \\
$a_{i}$ & 1.3881 & 1.3892 & 0.5647 \\
$b_{i}$ & 1.0943 & 0.9240 & - \\
$c_{i}$ & 1.7057 & 1.4620 & - \\
$d_{i}$ & 0.4421 & 0.4775 & - \\
\hline
\end{tabular}

but not to the energy of the system. The pressure becomes

$$
P_{r}=P+\rho_{B} \Sigma_{r}
$$

where the rearrangement self-energy, $\Sigma_{r}$, is given by

$$
\Sigma_{r}=\frac{\partial g_{N \omega}}{\partial \rho_{B}} \omega_{0} \rho_{B}-\frac{\partial g_{N \sigma}}{\partial \rho_{B}} \sigma \rho_{S}
$$

where $\rho_{S}$ is the scalar density. It can be verified that the contribution from the rearrangement self-energy restores the thermodynamical relation

$$
P_{r}=\rho_{B}^{2} \frac{\partial}{\partial \rho_{B}}\left(\frac{\epsilon}{\rho_{B}}\right),
$$

which is otherwise violated. Below we always consider the pressure including the rearrangement term and drop the subscript $r$.

\section{HYPERON-MESON COUPLING CONSTANTS}

To extend the description of matter to full baryon octet we need the hyperon-meson coupling constants. Because the information on the properties of hypernuclear matter is far less extensive than for nucleons their values can not be fixed with certainty and several approaches have been used in the literature.

One way to fix these couplings is to consider the $\mathrm{SU}(3)$ flavor symmetric model, namely the octet model. In this model the degrees of freedom are represented by the lowest non-trivial irreducible representation (IR) of the symmetry group which is physically possible, $\{8\}$, that is the baryon octet and the mesonic octet.

Let us consider first the interaction between the baryon octet, $J^{P}=\frac{1}{2}^{+}$, and the vector meson octet, $J^{P}=$ $1^{-}$. Within the assumption of $\mathrm{SU}(3)$-flavor symmetry, one can express the meson-baryon coupling constants in terms of only two parameters [59], the nucleon- $\boldsymbol{\rho}_{\mu}$ meson coupling constant $g_{N \rho}$ and the $F /(F+D)$ ratio of 
the vector octet $\alpha_{V}$ :

$$
\begin{aligned}
& g_{N \rho}=g_{8}, \quad g_{\Xi \rho}=-g_{8}\left(1-2 \alpha_{V}\right), \\
& g_{\Sigma \rho}=2 g_{8} \alpha_{V}, \quad g_{\Lambda \rho}=0, \\
& g_{N \omega_{8}}=\frac{1}{\sqrt{3}} g_{8}\left(4 \alpha_{V}-1\right), \quad g_{\Xi \omega_{8}}=-\frac{1}{\sqrt{3}} g_{8}\left(1+2 \alpha_{V}\right), \\
& g_{\Sigma \omega_{8}}=\frac{2}{\sqrt{3}} g_{8}\left(1-\alpha_{V}\right), \quad g_{\Lambda \omega_{8}}=-\frac{2}{\sqrt{3}} g_{8},\left(1-\alpha_{V}\right),
\end{aligned}
$$

where we have shown only the coupling constants relevant for our model. The vector-meson dominance model [60] predicts $\alpha_{V}=1$, which is a result of the universal coupling of the $\boldsymbol{\rho}_{\mu}$ meson to the isospin current. This result is also in agreement with recent QCD sum rules calculations [55]. Therefore, we set $\alpha_{V}=1$ in hyperon$\boldsymbol{\rho}_{\mu}$-meson coupling constants in Eq. (13), i.e.,

$$
g_{\Xi \rho}=g_{8}, \quad g_{\Sigma \rho}=2 g_{8}, \quad g_{\Lambda \rho}=0 .
$$

To describe the mixing between the singlet and the octet members of the vector nonet, one has to introduce two additional parameters, the flavor singlet coupling constant, $g_{1}$, and the vector mixing angle, $\theta_{V}$. Therefore, the coupling of the baryon to the physical $\omega_{\mu}$-meson state now reads

$$
g_{B \omega}=\cos \theta_{V} g_{1}+\sin \theta_{V} g_{B \omega_{8}} .
$$

It is widely accepted that the mixing between nonstrange and strange quark wave functions in the $\omega$ and $\phi$ mesons is ideal [61, 62]. In this limit $\tan \theta_{V}=1 / \sqrt{2}$ and assuming again $\alpha_{V}=1$ we obtain the baryon $-\omega_{\mu}$ meson couplings as

$$
\begin{aligned}
& \frac{g_{N \omega}}{\sin \theta_{V}}=\sqrt{2} g_{1}+\sqrt{3} g_{8}, \\
& \frac{g_{\Xi \omega}}{\sin \theta_{V}}=\sqrt{2} g_{1}-\sqrt{3} g_{8}, \\
& \frac{g_{\Sigma \omega}}{\sin \theta_{V}}=\frac{g_{\Lambda \omega}}{\sin \theta_{V}}=\sqrt{2} g_{1} .
\end{aligned}
$$

Next we assume that the nucleon does not couple to pure strange mesons $\left(\phi_{\mu}\right)$. One then obtains the relation between the singlet and the octet coupling constants [63]:

$$
g_{1}=\sqrt{6} g_{8},
$$

which allows one to eliminate $g_{1}$ from Eqs. (16). Thus, the hyperon- $\omega_{\mu}$-meson coupling constants can be related to the nucleon one

$$
g_{\Xi \omega}=\frac{1}{3} g_{N \omega}, \quad g_{\Sigma \omega}=g_{\Lambda \omega}=\frac{2}{3} g_{N \omega} .
$$

We are now left with the hyperon-scalar-meson $(\sigma)$ coupling constants. In order to fix these parameters, we consider the relations among the coupling constants for the scalar octet [54], which are given [in analogy with the relations for the vector meson octet in Eq. [13] ] by

$$
\begin{aligned}
& g_{N a_{0}}=g_{S}, \quad g_{\Xi a_{0}}=-g_{S}\left(1-2 \alpha_{S}\right), \\
& g_{\Sigma a_{0}}=2 g_{S} \alpha_{S}, \quad g_{\Lambda a_{0}}=0, \\
& g_{N \sigma_{8}}=\frac{1}{\sqrt{3}} g_{S}\left(4 \alpha_{S}-1\right), \quad g_{\Xi \sigma_{8}}=-\frac{1}{\sqrt{3}} g_{S}\left(1+2 \alpha_{S}\right), \\
& g_{\Sigma \sigma_{8}}=\frac{2}{\sqrt{3}} g_{S}\left(1-\alpha_{S}\right), \quad g_{\Lambda \sigma_{8}}=-\frac{2}{\sqrt{3}} g_{S}\left(1-\alpha_{S}\right) .
\end{aligned}
$$

The mixing between the singlet and the octet states of the scalar nonet is given by

$$
g_{B \sigma}=\cos \theta_{S} g_{1}+\sin \theta_{S} g_{B \sigma_{8}} .
$$

Then, the $\sigma$-meson-baryon coupling constants are

$$
\begin{aligned}
g_{N \sigma} & =\cos \theta_{S} g_{1}+\sin \theta_{S}\left(4 \alpha_{S}-1\right) g_{S} / \sqrt{3}, \\
g_{\Lambda \sigma} & =\cos \theta_{S} g_{1}-2 \sin \theta_{S}\left(1-\alpha_{S}\right) g_{S} / \sqrt{3}, \\
g_{\Sigma \sigma} & =\cos \theta_{S} g_{1}+2 \sin \theta_{S}\left(1-\alpha_{S}\right) g_{S} / \sqrt{3}, \\
g_{\Xi \sigma} & =\cos \theta_{S} g_{1}-\sin \theta_{S}\left(1+2 \alpha_{S}\right) g_{S} / \sqrt{3} .
\end{aligned}
$$

Combining the couplings, we obtain

$$
\begin{aligned}
g_{\Lambda \sigma}+g_{\Sigma \sigma} & =2 \cos \theta_{S} g_{1}, \\
g_{N \sigma}+g_{\Xi \sigma} & =2 \cos \theta_{S} g_{1}-2 \sin \theta_{S}\left(1-\alpha_{S}\right) g_{S} / \sqrt{3} \\
& =\cos \theta_{S} g_{1}+g_{\Lambda \sigma} .
\end{aligned}
$$

By eliminating $g_{1}$ from these expressions we obtain a relation

$$
2\left(g_{N \sigma}+g_{\Xi \sigma}\right)=3 g_{\Lambda \sigma}+g_{\Sigma \sigma},
$$

which is valid for arbitrary values of the four parameters $\alpha_{S}, g_{1}, g_{S}$ and $\theta_{S}$. In particular, it is satisfied for the values of the coupling constants in the $\mathrm{SU}(6)$ symmetric quark model. Indeed, the latter model assumes that the $\sigma$ meson is a pure up and down state, therefore for strangeness $-1 \Lambda$ and $\Sigma$ baryons $g_{\Lambda \sigma}=g_{\Sigma \sigma}=\frac{2}{3} g_{N \sigma}$, whereas for strangeness $-2 \Xi$ baryons $g_{\Xi \sigma}=\frac{1}{3} g_{N \sigma}$. Due to the constraint (27) the parameter space of our model is spanned by three out of the four parameters $\alpha_{S}, g_{1}$, $g_{S}$ and $\theta_{S}$. Further constraints on the parameter space can be placed because the hyperon coupling constants must be positive and less than the nucleon coupling constant. This implies two additional constraints, which can be translated into constraints on the range of variability of the hyperon-scalar-meson coupling constants. Indeed, by expressing one of the hyperon coupling constants, say $g_{\Xi \sigma}$, in terms of the others with the help of Eq. (27) one finds

$$
g_{\Xi \sigma}=\frac{1}{2}\left(3 g_{\Lambda \sigma}+g_{\Sigma \sigma}\right)-g_{N \sigma},
$$

and therefore, by requiring that

$$
0 \leq g_{\Xi \sigma} \leq g_{N \sigma},
$$


one is left with the following two simultaneous inequalities

$$
g_{N \sigma} \leq \frac{1}{2}\left(3 g_{\Lambda \sigma}+g_{\Sigma \sigma}\right) \leq 2 g_{N \sigma} .
$$

To explore the parameter space spanned by the hyperon$\sigma$ meson couplings we use our nuclear density functional and fix the remaining couplings. We fix the value of $g_{\Lambda \sigma}$ at the value provided by the Nijmegen soft core (NSC) hypernuclear potential model of Ref. [64] and vary the range of couplings $g_{\Sigma \sigma}$ within the limits provided by Eq. (30). The corresponding $g_{\Xi \sigma}$ couplings are found from Eq. (28). Analogously, to explore the range of admissible $g_{\Lambda \sigma}$ couplings, we next fix the value of $g_{\Sigma \sigma}$ to the value provided by the NSC model and change the $g_{\Lambda \sigma}$ coupling within a range which keeps the values of $g_{\Xi \sigma}$ consistent with Eq. (29). The parameter space is further limited by the requirement that the maximal values of $g_{\Lambda \sigma}$ and $g_{\Sigma \sigma}$ should be below their values in the $\mathrm{SU}(6)$ symmetric model. This latter constraint is motivated physically by our search for a stiff EoS of hypernuclear matter.

\section{RESULTS}

The matter in evolved compact stars is charge neutral and in $\beta$-equilibrium. These two conditions are imposed when computing the EoS at zero temperature. For young neutron stars at non-zero temperature a thermal ensemble of neutrinos should be included for temperatures $T \geq 5 \mathrm{MeV}$. In this case a common practice is to vary the lepton fraction of matter in a certain range compatible with astrophysical simulations. We treat these distinct cases in turn below.

\section{A. Zero temperature}

We start the discussion of our results by comparing the EoS of purely nucleonic matter, computed using DD parametrization of nuclear matter, to the EoS where interacting hyperons are added with couplings taken according to the SU(6) quark model; see Fig. 1. The appearance of hyperons, which is triggered by the fact that the cost of having a hyperon heavier than a nucleon is energetically more favorable than a neutron at the top of the Fermi sea, always softens the EoS. Because in our search the coupling constants are bound from above by their values in the $\mathrm{SU}(6)$ model, these two EoS correspond to the stiffest and softest EoS in our collection.

Our next step is to explore the impact of the variation of the hyperon-scalar-meson coupling on the EoS. As explained previously in Sec. IV we keep the hyperonvector-meson couplings fixed at the values given by Eqs. (14) and (18) and vary the hyperon-scalar-meson coupling constants in the range and manner defined above.

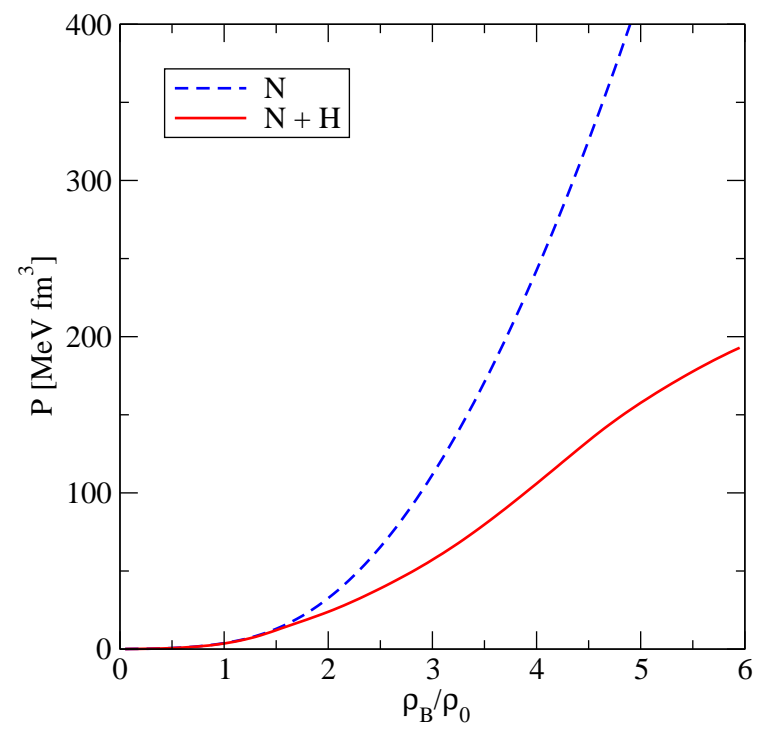

FIG. 1. (Color online) Equations of state of nuclear (dashed, blue online) and hypernuclear (solid, red online) matter. The coupling constants for nuclear matter correspond to densitydependent (DD) ME2 parametrization of Ref. [56]. The coupling constants of hyperons are related to the nucleonic ones according to the $\mathrm{SU}(6)$ symmetric quark model. The density is normalized to the nuclear saturation density $\rho_{0}=0.152$ $\mathrm{fm}^{-3}$ in the DD ME2 parametrization.

The left panel of Fig. 2 shows the dependence of the EoS of hypernuclear matter on variations of the coupling constant in the range $0.26 \leq x_{\Sigma \sigma} \leq 0.66$ at fixed $x_{\Lambda \sigma}=0.58$ (a value that corresponds to the NSC model), where $x_{H \sigma}=g_{H \sigma} / g_{N \sigma}$ is the ratio of the hyperon- $\sigma$ and the nucleon- $\sigma$ coupling constants. The resulting EoS covers the shaded area, which is bound by the two EoSs corresponding to the limiting values of the parameter $g_{\Sigma \sigma} ;$ in addition we show the special case where the $g_{\Sigma \sigma}$ parameter is fitted to reproduce the empirical value of the $\Sigma$-hyperon potential in nuclear matter at saturation $U_{\Sigma}=30 \mathrm{MeV}$ according to

$$
U_{\Sigma}=\frac{g_{\Sigma \sigma}}{g_{N \sigma}}\langle\sigma\rangle+\frac{g_{\Sigma \omega}}{g_{N \omega}}\langle\omega\rangle,
$$

where $\langle\ldots\rangle$ refers to the mean-field value of the field. The right panel of Fig. 2 2 shows the dependence of the EoS of hypernuclear matter on variations of the $g_{\Lambda \sigma}$ coupling constant in the range $0.52 \leq x_{\Lambda \sigma} \leq 0.66$ at fixed $x_{\Sigma \sigma}=$ 0.448 (as implied by the NSC model). The set of resulting EoS covers the shaded area; we also show explicitly the limiting cases as well as the special case, where the $g_{\Lambda \sigma}$ coupling is fitted to reproduce the $\Lambda$-hyperon potential in nuclear matter at saturation $U_{\Lambda}=-28 \mathrm{MeV}$ according to an equation analogous to Eq. (31). As expected, the reduction of the hyperon couplings stiffens the EoS. The stiffest hypernuclear EoS in this set corresponds to the values of couplings $x_{\Sigma \sigma}=0.448$ and $x_{\Lambda \sigma}=0.52$.

Figure 3 shows the particle fractions of fermions, de- 


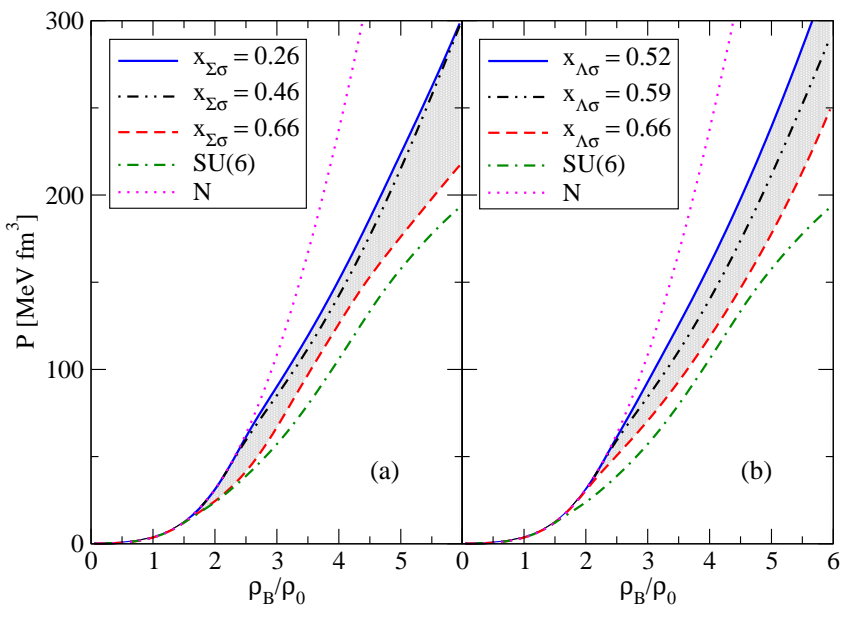

FIG. 2. (Color online) Equations of state of hypernuclear matter for a range of values of hyperon- $\sigma$-meson couplings defined in terms of $x_{H \sigma}=g_{H \sigma} / g_{N \sigma}, H \in \Lambda, \Sigma$. The nucleonic EoS (dotted line, magenta online) and hyperonic EoS with SU(6) quark model couplings (dot-dashed line, green online) are shown as a reference. The nucleonic coupling constants correspond to the DD-ME2 parametrization [56]; the hyperon-vector-meson couplings are fixed as explained in the text. In panel (a) we assume $x_{\Lambda \sigma}=0.58$, as in the NSC potential model, and a range $0.26 \leq x_{\Sigma \sigma} \leq 0.66$ which generates the shaded area; in (b) we assume $x_{\Sigma \sigma}=0.448$, as in the NSC potential model, and a range $0.26 \leq x_{\Lambda \sigma} \leq 0.66$. The cases $x_{\Sigma \sigma}=0.46$ (left panel) and $x_{\Lambda \sigma}=\overline{0.59}$ (right panel), shown by dash-double-dotted (black online) lines, fit the depth of the potentials of the $\Sigma^{-}$and $\Lambda$ hyperons in nuclear matter at saturation.

fined as the ratio of particle number densities to the baryon number density for the limiting cases of the $\mathrm{SU}(6)$ quark model couplings and the stiffest hypernuclear EoS. There are a number of features that are common to both EoSs. The appearance of $\Sigma^{-}$hyperons, which compensate for the positive change of the nuclear matter and excess negative isospin of the neutrons, is favored at low density. They contribute to the suppression and eventual extinction of electron and muon populations at large densities, i.e., the matter becomes deleptonized. Once the population of $\Sigma^{-}$hyperons reaches that of the protons and charge neutrality is established, its further increase is not favored. Instead, an excess of neutral $\Lambda$ hyperons builds up and dominates the hyperon population at asymptotically large densities. In the case of softer EoS (left panel of Fig. 3) the deleptonization and onset of hyperons like $\Sigma^{0,+}$ and cascades $\Xi^{0,-}$ occurs at densities lower than in the case of the stiff EoS (right panel of Fig. (3). This is the consequence of the fact that the large hyperon-meson couplings favor the formation of hyperons, which in turn soften the EoS. Thus the SU(6) quark model, with its large meson-hyperon couplings favors the onset of hyperons at low densities, which produces a soft EoS. Conversely, the stiffest EoS, which corresponds to

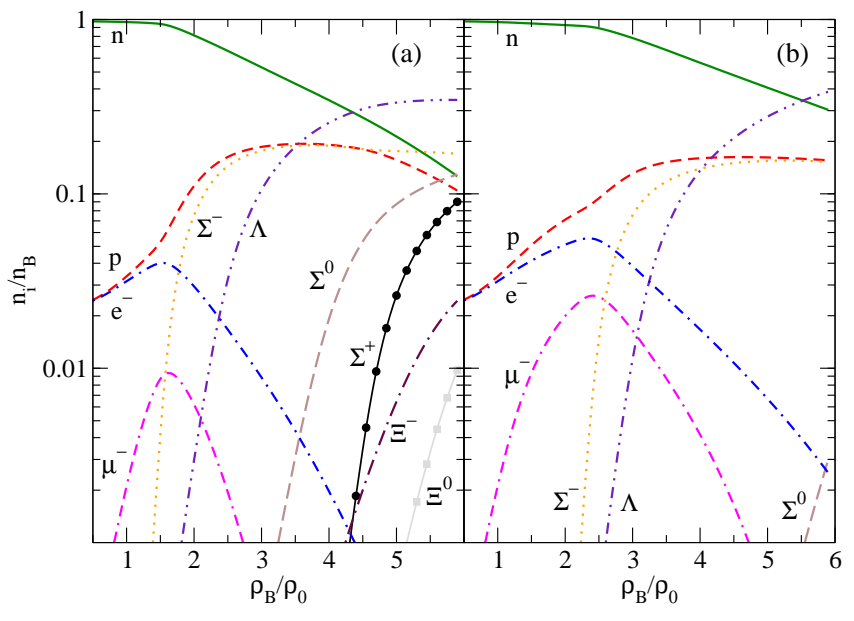

FIG. 3. (Color online) Particle fractions in hypernuclear matter at $T=0$ : (a) hyperon-scalar meson couplings are fixed as in the SU(6) symmetric quark model, and (b) a stiff hypernuclear EoS from our parameter space with $x_{\Sigma \sigma}=0.448$ and $x_{\Lambda \sigma}=0.52$.

small values of the hyperon-meson couplings, disfavors the onset of hyperons.

\section{B. Finite temperatures}

Compact stars become transparent to neutrinos after about a minute of formation in a supernova explosion. Prior to the neutrino-transparency era the star is hot, with temperatures on the order of several tens of $\mathrm{MeV}$, and neutrinos are in equilibrium with matter. The neutrino thermal distribution is characterized by the neutrino chemical potential $\mu_{\nu}$ and neutrino fraction $Y_{\nu}=n_{\nu} / n_{B}$, i.e., the ratio of the neutrino number density to the baryon number density. The neutrino fraction is commonly parameterized by specifying the lepton fraction for each flavor $Y_{L}=Y_{l}+Y_{\nu}$, where $Y_{l}$ is the fraction of the charged leptons of a given flavor. The relevant to proto-neutron stars range of lepton fraction is $0.1 \leq Y_{L} \leq 0.4$. Figure 4 shows the EoS of hot hypernuclear matter in the cases when there is a thermal population of neutrinos with $0.1 \leq Y_{L} \leq 0.4$ (shaded area) and in the absence of neutrinos, i.e., $Y_{\nu}=0$ and $Y_{l}$ determined from $\beta$-equilibrium with $\mu_{\nu, l}=0$, as in cold hypernuclear matter. The influence of including the neutrino population was studied for two EoS: a soft EoS with parameters according to the $\mathrm{SU}(6)$ quark model and a stiff EoS with $x_{\Sigma \sigma}=0.448$ and $x_{\Lambda \sigma}=0.52$ (see Fig. 2 for the $T=0$ counterparts). It can be seen that the EoS is stiff when neutrinos are present $\left(Y_{L}=0\right)$ and as the fraction of neutrinos, i.e., $Y_{L}$, increases the EoS becomes stiffer. The stiffening of the EoS can be attributed to the fact that the thermal population of neutrinos adds its contribution to the pressure of matter. However, neutri- 


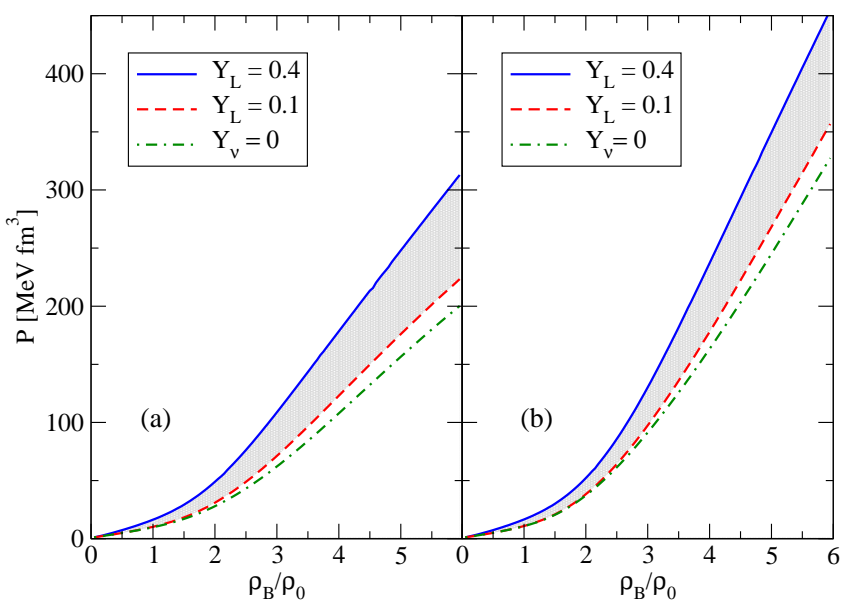

FIG. 4. (Color online) Equation of state in the DD-ME2 parametrization [56] at finite temperature, $T=50 \mathrm{MeV}$. (a) The hyperon-scalar-meson coupling constants are fixed by the quark model. In this figure the dependence on the presence of trapped neutrinos is shown. The dot-dashed line (green online) corresponds to the case without neutrinos. The presence of neutrinos make the EoS stiffer. The dashed region represents the variation of the EoS with the lepton fraction. The dashed line (red online) corresponds to a lepton fraction $Y_{L}=0.1$ and the full line (blue online) corresponds to $Y_{L}=0.4$; (b) same as left, in which the coupling constants are the one of the stiffest case considered, corresponding to $g_{\Sigma \sigma}=0.448 g_{N \sigma}$, fixed by the NSC potential and $g_{\Lambda \sigma}=0.52 g_{N \sigma}$.

nos stiffen the EoS also indirectly by changing the composition of matter. As seen in Figs. 5 and 6 the presence of neutrinos has a dramatic effect on charge leptons: The deleptonization effect observed in neutrino-less matter is reversed when neutrinos are present, which has the consequence that the $\Sigma^{-}$hyperons are not favored in neutrino-rich matter. In fact the sequence of appearance of charged $\Sigma$ hyperons is reversed (cf. the left and right panels of Fig. (5). Since neutrinos suppress the hyperon fractions (predominantly $\Lambda$ hyperon), at fixed baryon density, the EoS is closer to the nucleonic one, which as we have seen, is always stiffer than the hypernuclear EoS. By comparing Figs. 5 and 6 one can access how various choices of the hyperon-scalar-meson couplings influences the composition of matter. The general trend, observed at $T=0$ (Fig. 3), persists at non-zero temperatures, i.e., the thresholds for the onset of the various hyperons shift to higher densities for smaller values of the couplings of hyperons to the scalar mesons. The models with high values of these couplings are softer and more hyperonrich than the ones with smaller values of these couplings.

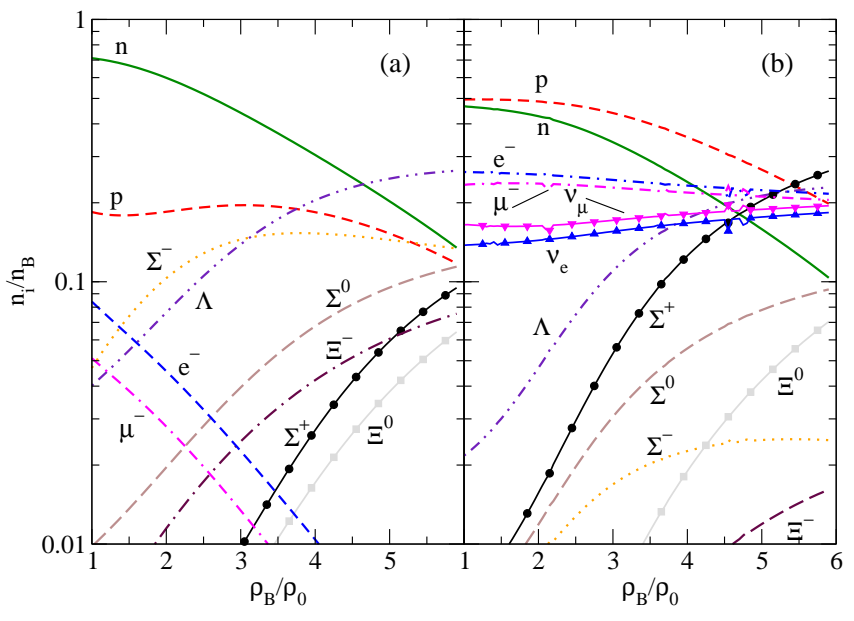

FIG. 5. (Color online) Particle fractions in hypernuclear matter at $T=50 \mathrm{MeV}$ for soft hypernuclear EoS based with couplings fixed by $\mathrm{SU}(6)$ symmetric quark model. In panel (a) $Y_{\nu}=0$, and in panel (b) $Y_{L}=0.4$

\section{Polytropic fits to the EoS}

Numerical relativity and astrophysics problems frequently require a simple parametrization of the EoS matter at zero temperature. The polytropic form of the EoS has been one of the common choices and more recently piecewise polytropic EoS were used to construct accurate representations of the microscopic EoS [65]. We use below the representation

$$
P=\sum_{i=1}^{4} K_{i} \rho^{\Gamma_{i}} \theta\left(\rho-a_{i} \rho_{0}\right) \theta\left(b_{i} \rho_{0}-\rho\right),
$$

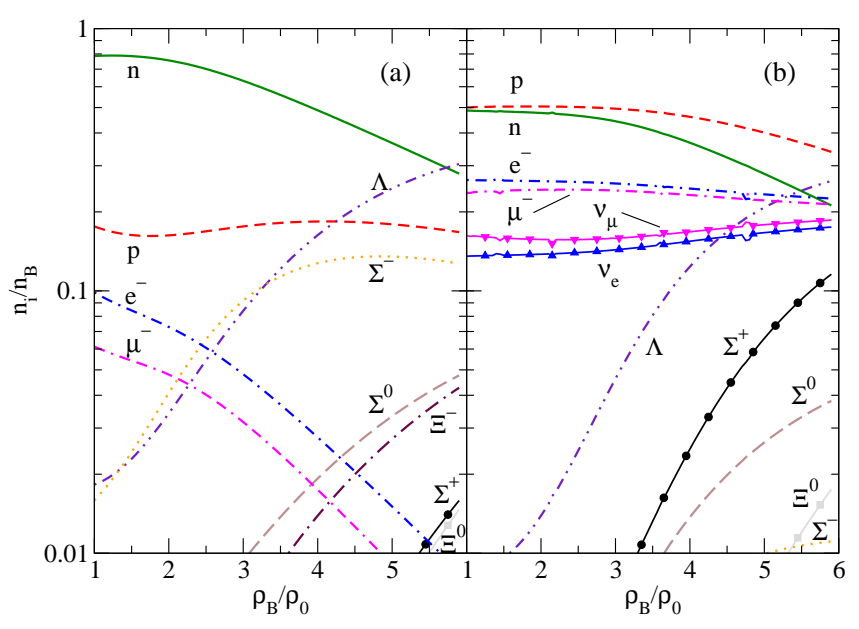

FIG. 6. (Color online) Same as in Fig. 5, but for a stiff hypernuclear EoS with to $x_{\Sigma \sigma}=0.448$ and $x_{\Lambda \sigma}=0.52$. 
TABLE II. Piecewise polytropic parametrization of various EoS according to Eq. (32). Set A corresponds to a pure nucleonic EoS. The hyperonic EoS with different values $x_{\Sigma \sigma}$ and $x_{\Lambda \sigma}$ are arranged as follows: set $\mathrm{B}, x_{\Lambda \sigma}=0.52$ and $x_{\Sigma \sigma}=0.448$; set $\mathrm{C}, x_{\Lambda \sigma}=0.66$ and $x_{\Sigma \sigma}=0.448$; set D, $x_{\Lambda \sigma}=0.58$ and $x_{\Sigma \sigma}=0.26$ and set $\mathrm{E}, x_{\Lambda \sigma}=0.58$ and $x_{\Sigma \sigma}=0.66$; Set $\mathrm{F}$ refers to the hyperonic EOS with $x_{Y Y \sigma}$ fixed to the values of the SU(6) symmetric quark model. The dimensionful constant $K_{i}$ is given in units of $\mathrm{MeV} \mathrm{fm}{ }^{3+3 \Gamma_{i}}$.

\begin{tabular}{|c|c|c|c|c|c|c|c|c|}
\hline$\imath$ & $K_{i}$ & $\Gamma_{i}$ & $a_{i}$ & $b_{i}$ & $K_{i}$ & $\Gamma_{i}$ & $a_{i}$ & $b_{i}$ \\
\hline & \multicolumn{4}{|c|}{ Set A } & \multicolumn{4}{|c|}{ Set B } \\
\hline 1 & 3.68535 & 2.86324 & 0.5 & 1.35 & 3.56837 & 3.12001 & 0.5 & 2.5 \\
\hline 2 & 3.73399 & 3.07344 & 1.35 & 3 & 7.66522 & 2.27431 & 2.5 & 3 \\
\hline 3 & 6.89825 & 2.54758 & 3 & 5 & 12.0670 & 1.86718 & 3 & 4 \\
\hline \multirow[t]{2}{*}{4} & 13.2029 & 2.16247 & 5 & 10 & 12.9132 & 1.81431 & 4 & 6 \\
\hline & \multicolumn{4}{|c|}{ Set $C$} & \multicolumn{4}{|c|}{ Set D } \\
\hline 1 & 3.76801 & 2.98366 & 0.5 & 2.25 & 3.55834 & 3.12437 & 0.5 & 2.5 \\
\hline 2 & 9.15775 & 1.86209 & 2.25 & 3 & 9.18876 & 2.08451 & 2.5 & 3 \\
\hline 3 & 9.64638 & 1.80967 & 3 & 5 & 12.9246 & 1.77419 & 3 & 5 \\
\hline \multirow[t]{2}{*}{4} & 7.79875 & 1.94152 & 5 & 6 & 15.7905 & 1.64781 & 5 & 6 \\
\hline & \multicolumn{4}{|c|}{ Set E } & \multicolumn{4}{|c|}{ Set $F$} \\
\hline 1 & 4.24406 & 2.50544 & 0.5 & 3.5 & 4.01772 & 2.62586 & 0.5 & 2 \\
\hline 2 & 9.27949 & 1.88033 & 3.5 & 4.25 & 5.49227 & 2.13420 & 2 & 4.25 \\
\hline 3 & 18.9055 & 1.38903 & 4.25 & 5 & 10.5763 & 1.68309 & 4.25 & 5 \\
\hline 4 & 25.6369 & 1.19845 & 5 & 6 & 24.7673 & 1.15400 & 5 & 6 \\
\hline
\end{tabular}

where $\Gamma_{i}$ is the polytropic exponent, $K_{i}$ is a dimensionful constant, and $\rho_{0}$ is the saturation density. These parameters are used to fit the EoS with a given interval of densities, the lower and upper boundaries specified by the parameters $a_{i}$ and $b_{i}$. The six different EoSs that were parameterized using Eq. (32) include a nuclear EoS, a hypernuclear EoS based on the $\mathrm{SU}(6)$ symmetric quark model, and four additional EoSs with $x_{\Sigma \sigma}$ and $x_{\Lambda \sigma}$ from the range discussed in the previous sections. The details are given in Table The polytropic representation above should be supplemented by a suitable EoS of matter in the crust of a neutron star below the density $\rho_{B} / \rho_{0} \leq 0.5$. This should be matched to given EoSs which are fitted only up to the crustal base, assumed to be at $0.5 \rho_{0}$. To optimize the fit, the density segments were chosen to optimally reflect the changes in the EoS; for example one knot, in the case of hypernuclear EoS, is chosen to be the density of the onset of hyperons. The change of the polytropic exponent with density is consistent with the expectation that as matter becomes more relativistic, the polytropic exponent decreases.

\section{Stellar structure}

The spherically symmetric solutions of Einstein's equations for self-gravitating fluids are given by the wellknown Tolman-Oppenheimer-Volkoff equations, which can be integrated for any given EoS. It is convenient

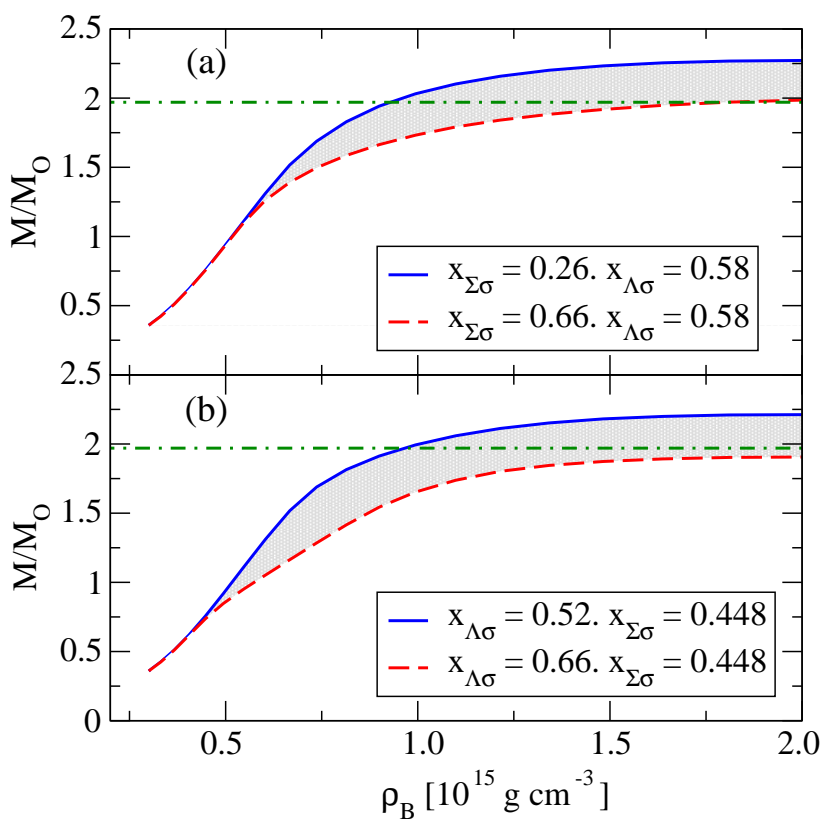

FIG. 7. (Color online) Dependence of the gravitational mass of compact hypernuclear stars on central density at zero temperature. The solid (blue online) and dashed (red online) show the limiting cases of parameter space as indicated in the panels (a) and (b). The dash-dotted (green online) line shows the observational lower limit on the maximum mass $1.97 M_{\odot}$.

to parametrize the equilibrium sequences of non-rotating configurations at zero temperature by their central density. A universal feature of these solutions is the existence of a maximum mass for any EoS; i.e., as the central density is increased a sequence reaches the configuration with the maximum mass and the stars with larger central densities are unstable towards gravitational collapse. A condition of stability for a sequence of configurations is $d M / d \rho_{c} \geq 0$; i.e., the mass should be an increasing function of the central density. Alternatively, the stability analysis of the lowest-order harmonics of pulsation modes (e.g., the fundamental radial pulsations) allows access to the stability of a configuration, for these are damped for stable stars and increase exponentially for unstable stars.

The range of considered hypernuclear EoS translates into the band of the stable configurations shown in Fig. 7. The hypernuclear configurations branch off from the purely nuclear configurations once the central density of a configuration reaches the threshold for appearance of hyperons in matter. The gravitational mass of hypernuclear stars increases with the density, indicating a stable branch of these objects, and reaches the maximum mass $\leq 2.25 M_{\odot}$ for densities of order $7 \rho_{0}$. Most of the sequences generated by the parameter space of the couplings considered is compatible with the observational bound $M / M_{\odot} \geq 1.97$. There is also room left for larger mass stars to allow for statistical distribution 


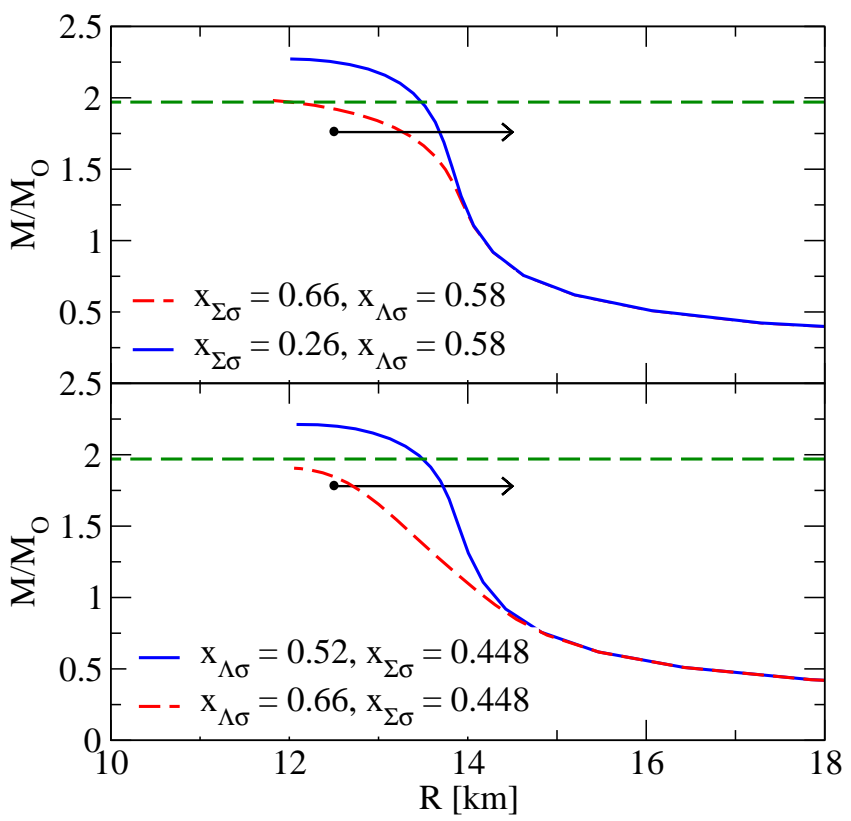

FIG. 8. (Color online) The mass-radius relations for compact hypernuclear stars at zero temperature. The labeling and parameter space is as in Fig. 7 The arrow shows the mass-radius constraint of Ref. [31] at $2 \sigma$ level, which is $M=1.76 M_{\odot}$ and $R \geq 12.5 \mathrm{~km}$.

of the masses of neutron stars beyond this limit. Note that $1.4 M_{\odot}$ canonical mass stars would be pure nucleonic for the softer subclass of EoS considered, whereas they would contain hypernuclear matter for the harder subclass; however all stars with $M>1.5 M_{\odot}$ contain hypernuclear matter.

In Fig. 8 we show the mass-radius relationship for the hypernuclear sequences. The configurations with masses close to the maximum mass $M \simeq 2 M_{\odot}$ have radii of $R \simeq 12 \mathrm{~km}$, whereas the canonical mass stars have radii of order $14 \mathrm{~km}$. The figure also shows the bound, which predicts that PSR J0437-4715, being a $M=1.76 M_{\odot}$ neutron star, has a radius $R>12.5 \mathrm{~km}$ at the $2 \sigma$ level. Clearly, the hypernuclear stars are consistent with this observation. Finally we note that it is not excluded that the low-mass neutron stars with $M \sim 1.2 M_{\odot}$ may already contain hypernuclear matter.

\section{CONCLUSIONS}

Despite decades of theoretical research on hypernuclear matter, the appearance of hyperons in compact stars remains an open issue. While the recent astrophysical measurements exclude a significant fraction of soft EoSs, the hyperonization of dense nuclear matter remains a seri- ous possibility. Our present study confirms this within a relativistic density functional approach to nuclear matter, where we investigated the impact of variation of the hyperon-scalar-meson couplings on the EoS of hypernuclear matter. The range of found EoS is sufficiently stiff to produce heavy compact stars $\left(M \leq 2.25 M_{\odot}\right)$. The radii of our sequences are located in the range of $12 \leq R \leq 14 \mathrm{~km}$. Piecewise polytropic fits for six representative EoSs are provided, which span the complete range of EoS from our parameter study.

The parameter space of couplings of hyperons to scalar mesons was explored, holding density-dependent nucleonic couplings fixed to their values suggested by the DD-ME2 parametrization of the nuclear density functional [56]. To allow for hyperonization in massive stars a requirement is to have small ratios of the hypernuclearto-nuclear couplings; in particular, hyperons need to be coupled to scalar mesons weaker than predicted by the $\mathrm{SU}(6)$ quark model.

By extending our studies to nonzero temperature and including thermal ensemble of neutrinos (present in a compact star during the first minute after birth) we confirm that the neutrinos stiffen the high-density EoS and impact the charge lepton content of hypernuclear matter. Instead of deleptonization with increasing density, seen in neutrino-less matter, the abundances of charged leptons remain constant, which has the consequence that the thresholds for appearance of charged $\Sigma$ 's is reversed.

The hyperonic abundances found in our EoS are broadly consistent with the predictions of other models, both early and recent, as these are determined by their mass spectrum and conditions of charge neutrality and $\beta$-equilibrium. By varying the scalar-meson couplings, we showed that the stiffer the EoS the larger are the densities at which any type of hyperon appears. For example, if for soft EoS a substantial amount of cascades can be built up, only trace amounts are found in stiff EoS. Furthermore, at non-zero temperatures the thresholds for the onset of hyperons are located at densities lower than those at zero temperature. It is evident that during the early cooling stage of a neutron star, i.e., the period where the temperature drops from tens of $\mathrm{MeV}$ to a $\mathrm{MeV}$ and matter becomes transparent to neutrinos, a rearrangement of particle content of matter must take place.

\section{ACKNOWLEDGMENTS}

We gratefully acknowledge the support by the HGSHIRe graduate program at Frankfurt University (G. C.) and a collaborative research grant of the Volkswagen Foundation (A. S.). We thank B. Friman, D. H. Rischke, P. Ring, and D. Vretrenar for discussions and R. G. E. Timmermans and S. Typel for useful communications. 
[1] M. Kramer, Proceedings of the International Astronomical Union, 291, 19 (2013), 1211.2457.

[2] P. B. Demorest, T. Pennucci, S. M. Ransom, M. S. E. Roberts, and J. W. T. Hessels, Nature (London) 467, 1081 (2010).

[3] J. Antoniadis, P. C. C. Freire, N. Wex, T. M. Tauris, R. S. Lynch, M. H. van Kerkwijk, M. Kramer, C. Bassa, V. S. Dhillon, T. Driebe, J. W. T. Hessels, V. M. Kaspi, V. I. Kondratiev, N. Langer, T. R. Marsh, M. A. McLaughlin, T. T. Pennucci, S. M. Ransom, I. H. Stairs, J. van Leeuwen, J. P. W. Verbiest, and D. G. Whelan. A Massive Pulsar in a Compact Relativistic Binary. ArXiv eprints (2013), 1304.6875.

[4] M. C. Miller, Nature (London) 467, 1057 (2010).

[5] J. R. Stone, P. A. M. Guichon, and A. W. Thomas, ArXiv e-prints (2010) 1012.2919.

[6] I. Vidaña, D. Logoteta, C. Providência, A. Polls, and I. Bombaci, EPL (Europhysics Letters) 94, 11002 (2011).

[7] C.-Y. Ryu, C. H. Hyun, and C.-H. Lee, Phys. Rev. C 84, 035809 (2011).

[8] L. Bonanno and A. Sedrakian, Astronomy \& Astrophysics 539, A16 (2012).

[9] M. Bejger, J. L. Zdunik, P. Haensel, and M. Fortin, Astron.\& Astrophys. 536, A92 (2011).

[10] S. Weissenborn, D. Chatterjee, and J. Schaffner-Bielich, Nuclear Physics A 881, 62 (2012).

[11] R. Lastowiecki, D. Blaschke, H. Grigorian, and S. Typel, ArXiv e-prints (2011), 1112.6430.

[12] S. Weissenborn, D. Chatterjee, and J. Schaffner-Bielich, Phys. Rev. C 85, 065802 (2012).

[13] M. Oertel, A. F. Fantina, and J. Novak, Phys. Rev. C 85, 055806 (2012).

[14] R. Mallick, ArXiv e-prints (2012), 1207.4872.

[15] K. Masuda, T. Hatsuda, and T. Takatsuka, Astrophys. J. 764, 12 (2013).

[16] C. H. Lenzi and G. Lugones, Astrophys. J. 759, 57 (2012).

[17] V. Dexheimer, J. Steinheimer, R. Negreiros, and S. Schramm, Phys. Rev. C 87, 015804 (2013).

[18] F. Gulminelli, A. R. Raduta, and M. Oertel, Phys. Rev. C 86, 025805 (2012), 1206.4924.

[19] W.-Z. Jiang, B.-A. Li, and L.-W. Chen, Astrophys. J. 756, 56 (2012), 1207.1686.

[20] F. Gulminelli, A.-R. Raduta, J. Margueron, P. Papakonstantinou, and M. Oertel, Journal of Physics, Conference Series 413, 012019 (2013), 1302.3776.

[21] J. L. Zdunik and P. Haensel, Aston. and Astophys. 551, A61 (2013), 1211.1231.

[22] P.-C. Chu and L.-W. Chen, ArXiv e-prints (2012), 1212.1388

[23] W.-Z. Jiang, R.-Y. Yang, and D.-R. Zhang, ArXiv eprints (2012), 1212.3686.

[24] C. Providência and A. Rabhi, Phys. Rev. C 87, 055801 (2013), 1212.5911.

[25] K. Masuda, T. Hatsuda, and T. Takatsuka, ArXiv eprints (2012), 1212.6803.

[26] F. Gulminelli, A. R. Raduta, M. Oertel, and J. Margueron, ArXiv e-prints (2013), 1301.0390.

[27] A. Sedrakian, PoS (Confinment X), 251 (2013), 1301.2675.
[28] S. Guillot, R. E. Rutledge, and E. F. Brown, Astrophys. J. 732, 88 (2011), 1007.2415.

[29] T. Güver, P. Wroblewski, L. Camarota, and F. Özel, Astrophys. J. 719, 1807 (2010), 1002.3825.

[30] D. K. Galloway and N. Lampe, Astrophys. J. 747, 75 (2012).

[31] S. Bogdanov, Astrophys. J. 762, 96 (2013).

[32] V. A. Ambartsumyan and G. S. Saakyan, Astronomicheski Zhurnal 37, 193 (1960).

[33] H. A. Bethe and M. B. Johnson, Nuclear Physics A 230, 1 (1974).

[34] S. A. Moszkowski, Phys. Rev. D 9, 1613 (1974).

[35] N. K. Glendenning, Physics Letters B 114, 392 (1982).

[36] N. K. Glendenning, Astrophys. J. 293, 470 (1985).

[37] N. K. Glendenning, Zeitschrift fur Physik A Hadrons and Nuclei 326, 57 (1987).

[38] N. K. Glendenning, Zeitschrift fur Physik A Hadrons and Nuclei 327, 295 (1987).

[39] F. Weber and M. K. Weigel, Nuclear Physics A 493, 549 (1989).

[40] J. I. Kapusta and K. A. Olive, Physical Review Letters 64, 13 (1990).

[41] J. Ellis, J. I. Kapusta, and K. A. Olive, Nuclear Physics B 348, 345 (1991).

[42] N. K. Glendenning and S. A. Moszkowski, Physical Review Letters 67, 2414 (1991).

[43] P. J. Ellis, R. Knorren, and M. Prakash, Physics Letters B 349, 11 (1995).

[44] J. Schaffner and I. N. Mishustin, Phys. Rev. C 53, 1416 (1996).

[45] F. Weber, Pulsars as astrophysical laboratories for nuclear and particle physics, (IOP Publishing, Bristol, 1999).

[46] A. Sedrakian, Progress in Particle and Nuclear Physics 58, 168 (2007).

[47] S. Balberg, I. Lichtenstadt, and G. B. Cook, Astrophys. J. Suppl. 121, 515 (1999).

[48] H.-J. Schulze and T. Rijken, Phys. Rev. C 84, 035801 (2011).

[49] D. Logoteta, I. Vidaña, C. Providência, A. Polls, and I. Bombaci, Journal of Physics Conference Series 342, 012006 (2012).

[50] I. L. Vartanian and N. K. Ovakimova, Soviet Astron. 21, 727 (1977).

[51] J. A. Pons, S. Reddy, M. Prakash, J. M. Lattimer, and J. A. Miralles, Astrophys. J. 513, 780 (1999).

[52] I. Bednarek, M. Kȩske, and R. Mańka, International Journal of Modern Physics D 13, 215 (2004).

[53] G. F. Burgio, H.-J. Schulze, and A. Li, Phys. Rev. C 83, 025804 (2011).

[54] G. Erkol, R. G. E. Timmermans, M. Oka, and T. A. Rijken, Phys. Rev. C 73, 044009 (2006).

[55] G. Erkol, R. G. E. Timmermans, and T. A. Rijken, Phys. Rev. C 74, 045201 (2006).

[56] G. A. Lalazissis, T. Nikšić, D. Vretenar, and P. Ring, Phys. Rev. C 71, 024312 (2005).

[57] S. Typel and H. H. Wolter, Nuclear Physics A 656, 331 (1999).

[58] C. Fuchs, H. Lenske, and H. H. Wolter, Phys. Rev. C 52, 3043 (1995). 
[59] J. J. de Swart, Reviews of Modern Physics 35, 916 (1963).

[60] J. J. Sakurai, Annals of Physics 11, 1 (1960).

[61] KLOE Collaboration, F. Ambrosino, A. Antonelli, S. Bertolucci, C. Bini, C. Bloise, S. Bocchetta, F. Bossi, P. Branchini, G. Capon, et al., Journal of High Energy Physics 7, 105 (2009).
[62] A. Kucukarslan and U.-G. Meißner, Modern Physics Letters A 21, 1423 (2006).

[63] C. B. Dover and A. Gal, Progress in Particle and Nuclear Physics 12, 171 (1984).

[64] P. M. M. Maessen, T. A. Rijken, and J. J. de Swart, Phys. Rev. C 40, 2226 (1989).

[65] J. S. Read, B. D. Lackey, B. J. Owen, and J. L. Friedman, Phys. Rev. D 79, 124032 (2009). 